\title{
PERFIL SOCIOECONÔMICO E DEMOGRÁFICO DE PUÉRPERAS ADOLESCENTES*
}

Sonia Maria Könzgen Meincke², Mayza Raphaella Pontes de Oliveira² ${ }^{2}$, Débora Raquel Soares Guedes Trigueiro ${ }^{3}$, Telma Elisa Carraro ${ }^{4}$, Emilainne Trindade Cavalcanti Gondim ${ }^{2}$, Neusa Collet ${ }^{5}$

RESUMO: Objetivou-se descrever o perfil socioeconômico e demográfico de adolescentes internadas na enfermaria de puerpério de um hospital universitário da Parába. Estudo quantitativo realizado de dezembro de 2008 a novembro de 2009, por meio de questionário estruturado, com 85 puérperas adolescentes. As principais variáveis analisadas foram: idade, cor, ocupação, estado civil, grau de escolaridade, renda familiare apoio recebido pela puérpera. Para análise foi utilizado o programa Statistical Package for the Social Sciences. Os resultados indicam que as adolescentes são de cor morena (69,4\%), com baixo nível de escolaridade (61,2\%), alto índice de evasão escolar (73,8\%), fora do mercado de trabalho $(92,9 \%)$, dependentes economicamente $(89,4 \%)$ e pertencentes às classes econômicas menos favorecidas $(85,9 \%$ ). Esta realidade contribui para a manutenção do ciclo da pobreza. A gravidez não planejada na adolescência gera consequências para a adolescente, sua família e sociedade. Destaca-se a importância do apoio familiar e social como fatores protetores.

PALAVRAS-CHAVE: Gravidez na adolescência; Perfil de saúde; Família; Enfermagem.

\section{SOCIO-ECONOMIC AND DEMOGRAPHIC PROFILE OF ADOLESCENT MOTHERS}

ABSTRACT: The objective was to describe the socio-economic and demographic profile of adolescents in the maternity ward of a university hospital in Paraíba. This quantitative study was carried out between December 2008 and November 2009, by means of a structured questionnaire given to 85 adolescent expectant mothers. The principle variables analyzed were: age, colour, occupation, marital status, educational level, family income and the support the expectant mother received. The program Statistical Package for the Social Sciences was used for the analysis. The results indicate that the adolescents are dark-skinned (69.4\%), with low levels of schooling (61.2\%) and high levels of truancy (73.8\%) and unemployment $(92.9 \%)$. They are economically dependent $(89.4 \%)$, and belong to less-favoured economic classes. This reality contributes to the continuation of the cycle of poverty. Unplanned pregnancy in adolescence creates consequences for the adolescent, her family and society. The importance of family and social support as protective factors was clear.

KEYWORDS: Pregnancy in adolescence; Health profile; Family: Nursing.

\section{PERFIL SOCIOECONÓMICO Y DEMOGRÁFICO DE PUÉRPERAS ADOLESCENTES}

RESUMEN: El objetivo fue describir el perfil socioeconómico y demográfico de adolescentes internadas en la enfermería de puerperio de un hospital universitario de Paraíba. Estudio cuantitativo realizado de diciembre de 2008 a noviembre de 2009, por medio de cuestionario estructurado, con 85 puérperas adolescentes. Las principales variables analizadas fueron: edad, color, ocupación, estado civil, grado de escolaridad, renta familiary apoyo recebido. Para análisis fue utilizado el programa Statistical Package for the Social Sciences. Los resultados apuntan que las adolescentes son de color moreno (69,4\%), con nivel bajo de escolaridad (61,2\%), elevado índice de evasión escolar (73,8\%), no están en el mercado de trabajo $(92,9 \%)$, son dependientes economicamente (89,4\%) y pertenecentes a las clases económicas menos favorecidas $(85,9 \%)$. Esta realidad contribuye para la manutención del ciclo de la pobreza. La gravidezno planeada en la adolescencia genera consecuencias para la adolescente, su familia y sociedad. Se destaca la importancia del apoyo familiar y social como factores protectores. PALABRAS-CLAVE: Gravidez en la adolescencia; Perfil de salud; Familia; Enfermería.

*Recorte da pesquisa multicêntrica "Redes Sociais de Apoio a Paternidade na Adolescência - RAPAD", financiada pelo CNPq Edital MCT/CNPq/MS-SCTIE-DECIT/CT - Saúde n. 022/2007.

${ }^{1}$ Enfermeira. Doutora em Enfermagem. Professora do Departamento e do Programa de Pós-Graduação em Enfermagem da Faculdade de Enfermagem da Universidade Federal de Pelotas - UFPel. Coordenadora da pesquisa multicêntrica RAPAD Rio Grande do Sul.

${ }^{2}$ Enfermeira.

${ }^{3}$ Enfermeira. Mestranda pelo Programa de Pós-Graduação em Enfermagem da Universidade Federal da Paraíba.

${ }^{4}$ Enfermeira. Pós-Doutora em Enfermagem. Professora do Departamento e do Programa de Pós-Graduação em Enfermagem da Universidade Federal de Santa Catarina. Líder do Grupo de Pesquisa Cuidando e Confortando - C\&C.

${ }^{5}$ Enfermeira. Doutora em Enfermagem. Professora do Departamento e do Programa de Pós-Graduação em Enfermagem da Universidade Federal da Paraíba. Líder do Grupo de Estudos e Pesquisa em Saúde da Criança e do Adolescente - GEPSCA.

Universidade Federal da Paraíba

Caixa Postal 141 - 58310-000 - Cabedelo-PB-Brasil

E-mail: neucollet@gmail.com

Cogitare Enferm. 2011 Jul/Set; 16(3):486-91 


\section{INTRODUÇÃO}

A ocorrência de uma gravidez na adolescência era considerada um evento comum e até mesmo esperado em décadas passadas; mais recentemente vem sendo concebida como problema de saúde pública que tem mobilizado tanto a sociedade civil como trabalhadores e pesquisadores da área da saúde para conhecer os determinantes de sua ocorrência ${ }^{(1-4)}$. Nesse contexto, a educação sexual de alunos adolescentes passa a fazer parte das obrigações dos educadores, além da família. Contudo, o despreparo e a insegurança para abordar a temática, preconceitos e tabus presentes em pais e professores, são geradores de barreiras para a realização de estratégias que garantam a formação da sexualidade sadia e a promoção da saúde dos adolescentes ${ }^{(5)}$.

A gestação na adolescência é um fenômeno com repercussões significativas para o indivíduo e para a sociedade. Para a adolescente, a gravidez precoce pode marcar e alterar toda a sua vida. Pela perspectiva da comunidade e do governo, esse fenômeno tem uma forte associação com baixos níveis educacionais e um impacto negativo no seu potencial de ascensão econômica ${ }^{(3)}$ bem como, na geração de mão-de-obra qualificada para os serviços, que são cada vez mais especializados.

Dentre os problemas relacionados à gravidez na adolescência encontram-se os pessoais, os familiares e os socioeconômicos ${ }^{(4)}$. A chance de evasão escolar, com consequente piora da qualificação profissional, e a tendência a proles numerosas, criam um ciclo de manutenção da pobreza ${ }^{(6)}$. Há, portanto, necessidade de avaliação quantitativa e qualitativa da questão, principalmente nos países em desenvolvimento, para verificação da necessidade da adoção de medidas pertinentes à sua prevenção, direcionando-as aos grupos mais vulneráveis ${ }^{(7)}$.

Frente ao exposto, salienta-se a importância de conhecer o perfil dessa população para a promoção de ações em saúde. A questão orientadora deste estudo foi: qual o perfil socioeconômico e demográfico das adolescentes internadas na enfermaria de puerpério de um hospital de referência para esse atendimento?

Acredita-se que, conhecendo profundamente esse perfil, poder-se-á ter elementos concretos para que as equipes de saúde de todos os níveis de atenção reflitam acerca de seu processo de trabalho na atenção à essas adolescentes a fim de viabilizar a construção de um projeto de educação em saúde que atenda às suas necessidades singulares. Para tanto, o objetivo desta pesquisa foi traçar o perfil socioeconômico e demográfico de adolescentes internadas na enfermaria de puerpério de um Hospital Universitário da Paraíba.

\section{METODOLOGIA}

Estudo descritivo com abordagem quantitativa e que consiste num recorte da pesquisa multicêntrica intitulada "Redes sociais de apoio à paternidade na adolescência-RAPAD" (8) desenvolvida entre três universidades públicas federais, a saber, Universidade Federal de Pelotas (UFPel - coordenação geral do projeto), Universidade Federal de Florianópolis (UFSC) e Universidade Federal da Paraíba (UFPB). Para o recorte deste artigo, os dados ora apresentados referem-se aqueles aos coletados na UFPB.

O projeto foi aprovado pelo Comitê de Ética em Pesquisa da Faculdade de Odontologia da UFPel, tendo obtido parecer favorável (Protocolo n. 007/2008), com ciência e autorização do Comitê de Ética do hospital em estudo. Aos sujeitos da pesquisa foram garantidos todos os direitos previstos na Resolução n. 196/96 do Conselho Nacional de Saúde ${ }^{(9)}$. Os responsáveis pelas puérperas adolescentes assinaram o Termo de Consentimento Livre e Esclarecido.

Os sujeitos do estudo foram todas as puérperas adolescentes, com idade inferior a 20 anos, que tiveram seus partos no referido hospital, no período de 03 de dezembro de 2008 a 02 de dezembro de 2009. Foram excluídas da pesquisa as puérperas que apresentaram dificuldades de comunicação, doenças maternas graves e/ou tiveram óbito fetal.

Para a coleta de dados foi aplicado um questionário estruturado, com questões fechadas e abertas, validado pela equipe do projeto. As variáveis analisadas incluíram: idade em número de anos completos; cor/raça; profissão e idade do início do trabalho; tipo de vínculo: formal ou não; estado civil; com quem reside; escolaridade em anos de escola concluídos; renda mensal e renda familiar (em reais); e apoio (se recebeu ou não, e quem a apoiou). Além do questionário aplicado pelas pesquisadoras, foram consultados os prontuários das adolescentes.

Para a sistematização e análise dos dados foi utilizado o Programa Statistical Package for the Social Sciences (SPSS). Cada questionário recebeu uma codificação, para manter o sigilo acerca do sujeito da pesquisa, e que será usada na apresentação dos resultados da seguinte forma: letra A, referindo-se à adolescente, e o respectivo código da entrevista. Os indicadores referentes aos dados socioeconômicos foram analisados utilizando-se tratamento estatístico com números relativos e absolutos, sendo apresentados sob a forma de tabelas e gráfico. 


\section{RESULTADOS}

O total de puérperas entrevistadas nesse estudo foi de 85, sendo 2(2,4\%) com 13 anos, 3(3,5\%) com 14 anos, 9(10,6\%) com 15 anos, 11(12,9\%) com 16 anos, 14(16,5\%) com 17 anos, 19(22,4\%) com 18 anos e 27(31,8\%) com 19 anos. A média de idade das puérperas adolescentes foi de 18,2 anos. Foi possível observar o predomínio de puérperas de cor morena, representando $69,4 \%$, seguida pelas de cor branca $(23,5 \%)$ e as de cor negra $(7,1 \%)$. Porém, não houve diferenças significativas de cor entre as faixas etárias.

Com relação ao estado civil, $71(83,5 \%)$ das puérperas adolescentes referiram estar casadas ou com companheiro e as outras $14(16,5 \%)$ referiram estar solteiras. Das 71 casadas, apenas 1(1,4\%) estava casada ou vivendo com companheiro que não era o pai de seu filho. Das 14 solteiras, 7(50\%) não possuíam vínculo com o pai de seu filho (o vínculo constituiu-se apenas durante a gravidez), 6(42,8\%) mantinham vínculo esporádico e $1(7,2 \%)$ não possuía vínculo porque o pai de seu filho havia falecido.

Quanto à moradia, apenas uma referiu morar sozinha $(1,2 \%)$ e, das $84(98,8 \%)$ que referiram não morar sozinhas, $30(35,7 \%)$ moravam apenas com o companheiro; $8(9,5 \%)$ moravam apenas com companheiro e filhos; e 46(54,8\%) constituíam modelos de famílias não nucleares ou extensas, das quais fazem parte irmãos, cunhados, sobrinhos, avós e sogros.

Todas as puérperas adolescentes entrevistadas referiram saber ler. Porém, 61 delas $(71,8 \%)$ relataram ter parado de estudar. Dentre os motivos para não continuar os estudos, houve prevalência da gravidez como causa de abandono escolar, em $73,8 \%$ das justificativas. Dentre as demais, os motivos foram: falta de interesse (A, 3007); não gostar de estudar (A, 3712); preguiça de ir às aulas (A, 3003); porque o companheiro não quer (A, 3017); por ter terminado o ensino médio (A, 3813; A, 3808; A, 3312; A, 3708; A, 3008; A, 3705; A, 3603; A, 3006; A, 3001; A, 3319; A, 3320; A, 3311; A, 3312).

Em relação ao nível de escolaridade, $33(38,8 \%)$ puérperas encontravam-se cursando o ensino médio e a maioria $(61,2 \%)$ delas encontrava-se cursando o ensino fundamental, apesar de ter idade para estar no ensino médio, algumas podendo até já o terem concluído.

Quanto aos anos completos de estudo: 1(1,3\%) tinha dois anos completos de estudo, $1(1,3 \%)$ tinha três anos, $2(2,6 \%)$ tinham quatro anos, $7(8,2 \%)$ tinham cinco anos, $13(15,3 \%)$ tinham seis anos, $9(10,6 \%)$ tinham sete anos, $19(22,4 \%)$ tinham oito anos, 5(5,9\%) tinham nove anos, 11(12,9\%) tinham dez anos, e 17(20\%) tinham onze anos de estudo.

A gravidez entre adolescentes pode levar ao afastamento do mercado de trabalho. Neste estudo, a maioria (92,9\%, 79 puérperas) não estava trabalhando e dentre as $6(7,1 \%)$ que trabalhavam, cada uma começou a trabalhar com as seguintes idades: $8,10,12,16,17 \mathrm{e}$ 18 anos. Apenas uma (1,3\%) tinha vínculo formal de trabalho. Nesse contexto, a principal fonte de renda das adolescentes foi a renda do companheiro $(56,5 \%) \mathrm{e}$, em seguida, a renda familiar (32,9\%), incluindo pai e/ou mãe da adolescente e irmãos. Apenas $9(10,7 \%)$ possuíam sua própria fonte de renda, oriunda de vínculo empregatício informal $(5,9 \%)$, de bolsa-escola $(2,4 \%)$ ou de pensão $(2,4 \%)$. Salienta-se que uma das adolescentes, que referiu trabalhar, não possuía renda, pois trabalha na roça para o próprio sustento. De acordo com a tabela 1 podemos observar que a renda mensal é variada, porém, com concentração maior entre aquelas sem renda, já que a maioria delas não trabalha.

Tabela 1- Renda mensal de puérperas adolescentes. João Pessoa, 2008/2009

\begin{tabular}{lcc}
\hline Renda mensal em R\$ & n & $\mathbf{\%}$ \\
\hline zero & 76 & 89,4 \\
50,00 & 1 & 1,2 \\
100,00 & 2 & 2,4 \\
280,00 & 1 & 1,2 \\
465,00 & 1 & 1,2 \\
500,00 & 1 & 1,2 \\
511,00 & 2 & 2,4 \\
$1.700,00$ & 1 & 1,2 \\
\hline Total & 85 & 100 \\
\hline
\end{tabular}

Fonte: Pesquisa RAPAD 2008/2009.

O número de pessoas que depende da renda da família da mãe adolescente pode ir além do número de pessoas que mora com a adolescente (pois outros indivíduos que não residem na mesma casa dependem dessa renda) ou ser até inferior, quando nem todos que moram na casa dependem da mesma renda. Pode-se observar, na tabela 2 , que na maioria $(51,8 \%)$ das famílias das adolescentes apenas três pessoas dependiam da renda familiar. No entanto, encontraram-se famílias em que até 11 pessoas dependiam da mesma renda.

O Novo Critério Padrão de Classificação Econômica/ Brasil ${ }^{(10)}$ é um sistema de pontuação padronizado e eficiente estimador da capacidade de consumo da população. Para isso, utiliza-se de informações objetivas com relação à 
posse de itens/produtos e ao grau de instrução do chefe da família. Por meio do conhecimento desses dados é possível estabelecer em que classe socioeconômica está inserida a família, pois esse critério conta com uma tabela que esta-

Tabela 2- Número de pessoas que dependem da renda familiar da mãe adolescente. João Pessoa, 2008/2009

\begin{tabular}{ccc}
\hline $\begin{array}{c}\mathrm{N}^{\mathrm{o}} \text { de pessoas que dependem } \\
\text { da renda familiar }\end{array}$ & $\mathrm{n}$ & $\%$ \\
\hline 2 & 2 & 2,4 \\
3 & 44 & 51,8 \\
4 & 10 & 11,8 \\
5 & 13 & 15,3 \\
6 & 8 & 9,4 \\
7 & 5 & 5,9 \\
9 & 1 & 1,2 \\
10 & 1 & 1,2 \\
11 & 1 & 1,2 \\
\hline Total & 85 & 100,0 \\
\hline
\end{tabular}

Fonte: Pesquisa RAPAD 2008/2009

belece pontos de corte para segmentação em classes, que variam de $\mathrm{A} 1 \mathrm{a}$ E. De acordo com esse novo critério foram obtidos resultados conforme apresentados no gráfico 1 .

Nas famílias das mães adolescentes, o chefe da família, na maioria das vezes, foi representado pela figura masculina; em 43(50,6\%) famílias o companheiro apareceu como chefe da família, em 14(16,5\%) o pai, em $8(9,4 \%)$ o sogro, em 3(3,5\%) o avô e em 1(1,2\%) o padrasto. Em 13(15,3\%) a mãe apareceu como chefe da família, em $2(2,4 \%)$ a sogra e em apenas $1(1,2 \%)$ a própria adolescente.

Gráfico 1 - Classificação socioeconômica das puérperas adolescentes. João Pessoa, 2008/2009

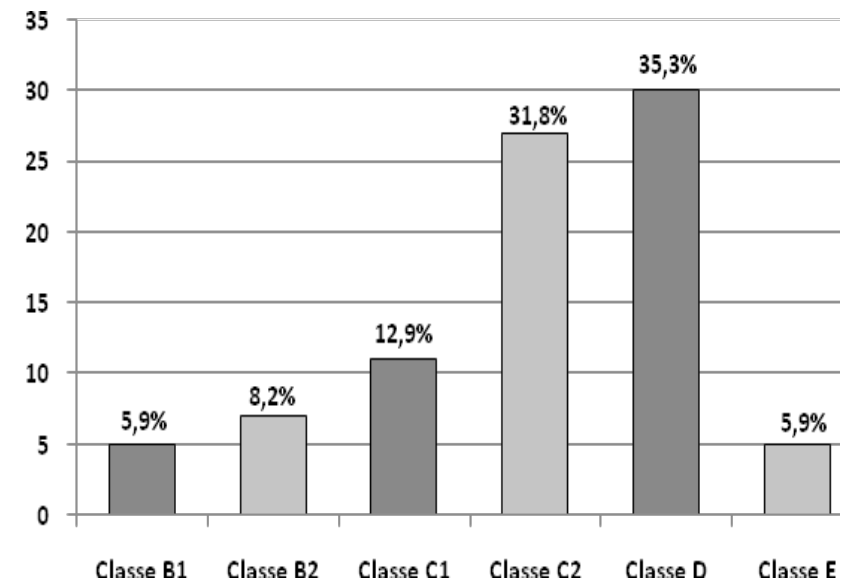

Fonte: Pesquisa RAPAD 2008/2009.
O nível de escolaridade dos chefes da família foi um dos critérios utilizados para classificação das puérperas adolescentes quanto à classe econômica, observandose os seguintes resultados: em $47,1 \%$ das famílias o chefe possuía apenas o ensino fundamental, em 32,9\% possuía o ensino médio, em $14,2 \%$ nunca estudou, e em apenas 3,5\% o chefe da família concluiu o ensino superior; e em 2,4\% algum curso técnico. A família é muito importante nessa fase da vida da adolescente, por se constituir em um grupo de apoio, oferecendo auxílio material e afetivo capaz de gerar efeitos positivos para que a adolescente tenha condições de enfrentar as mudanças típicas de uma gravidez precoce.

Todas as puérperas adolescentes relataram ter recebido algum tipo de apoio durante a gravidez, cuja fonte foi: $73(85,9 \%)$ pai de seu filho; $1(1,2 \%)$ companheiro que não é o pai de seu filho; $77(90,6 \%)$ mãe; $54(63,5 \%)$ pai; $4(4,7 \%)$ padrasto; $1(1,2 \%)$ madrasta; $41(48,2 \%)$ avó; 32(37,6\%) avô; 56(65,9\%) irmãos; 47 $(55,3 \%)$ primos; $53(62,4 \%)$ tia; $47(55,3 \%)$ tio; $53(62,4 \%)$ amigos; 53(62,4\%) sogra; 44(51,8\%) sogro; e 7(8,2\%) outras pessoas como cunhados e vizinhos.

\section{DISCUSSÃO}

As características sociais das puérperas adolescentes apresentam similaridade com dados encontrados em outras pesquisas, principalmente com relação à média de idade de 18,2 anos e a cor da pele parda ou morena ${ }^{(11)}$. A maioria das puérperas adolescentes entrevistadas estava casada ou vivia com companheiro. Porém, 54,8\% delas constituíam modelos de famílias não nucleares ou extensas, resultado da reorganização familiar devido à dependência financeira da puérpera adolescente e de seu companheiro.

Observa-se que a gravidez na adolescência fez com que surgissem novos arranjos familiares, nos quais as adolescentes passam a morar com o companheiro na casa de seus pais, ou vão morar com o companheiro na casa dos pais dele. Revela-se assim, que a gravidez na adolescência pode levarà desorganização familiar. Dessa forma, vários núcleos familiares passam a conviver num mesmo espaço físico, compondo e dividindo a renda e a organização familiar ${ }^{(3)}$.

A gravidez na adolescência foi referida pela maioria das puérperas entrevistadas como o motivo do abandono escolar, corroborando outro estudo ${ }^{(12)}$. Portanto, a maternidade precoce tem sido identificada como um dos fatores de afastamento e de dificuldade nos estudos entre adolescentes.

O alto índice de evasão escolar observado nesta pesquisa $(71,8 \%)$, associado ao afastamento do mercado de 
trabalho, traz graves consequências para a adolescente e seu filho, bem como para a sociedade em geral, principalmente porque, nessa faixa etária, uma das poucas opções de inserção social e de ascensão econômica se dá por intermédio do sistema educacional ${ }^{(3)}$.

Os anos de estudos podem refletir o nível de educação ou instrução do indivíduo. Pesquisas evidenciam que, quanto menor o número de anos de escolaridade, maior é a incidência de gestações entre adolescentes, ou seja, o grau de escolaridade está vinculado ao índice de gravidez em menores de 20 anos $^{(5,12)}$. Tal aspecto também foi identificado no presente estudo, pois as puérperas possuíam baixo nível de escolaridade e poucos anos completos de estudo.

Outras pesquisas mostram uma relação inversa entre o nível de escolaridade e a ocorrência de gravidez na adolescência, com o declínio de $40,7 \%$ entre as adolescentes não letradas para, praticamente zero, entre as que tinham 12 ou mais anos de estudo ${ }^{(4,13)}$.

A maternidade precoce implica, muitas vezes, em mães com menor grau de instrução, aumentando a probabilidade de levar a criança a contrair doenças infectocontagiosas, gastrintestinais, acidentais, entre outras. As consequências são: risco aumentado de morbimortalidade no primeiro ano de vida, além de pior rendimento escolar no futuro ${ }^{(12)}$. A escola tem papel fundamental na educação sexual de adolescentes no que se refere ao desenvolvimento da sexualidade saudável, livre dos riscos de DST/Aids e da gravidez indesejada e/ou precoce ${ }^{(5)}$.

Essas garotas, com baixa escolaridade, têm menor chance frente a um mercado de trabalho cada vez mais competitivo e, mesmo quando conseguem colocação, isso se dá em atividades com baixa remuneração, ou que as mantêm, frequentemente, na dependêmcia dos familiares ou companheiro, podendo criar um ciclo de manutenção da pobreza ${ }^{(6)}$.

O Ministério da Educação (ME) estabelece o limite etário para escolaridade de ensino fundamental de 6 a 14 anos e, para o ensino médio, de 15 a 18 anos. Sendo assim, a maioria das adolescentes entrevistadas deveria estar cursando o ensino médio, já que $94,2 \%$ (80) delas tinha 15 anos ou mais ${ }^{(14)}$. Contudo, neste estudo, a concentração ficou no ensino fundamental, evidenciando que as adolescentes estavam fora das fases recomendadas pelo recomendado pelo ME.

De acordo com Novo Critério Padrão de Classificação Econômica/Brasil ${ }^{(10)}$, a maioria das puérperas adolescentes desta pesquisa pertencia às classes $\mathrm{C} 2 \mathrm{e} \mathrm{D}$, classes essas mais desfavorecidas economicamente. Nenhuma das entre- vistadas pertencia às classes $\mathrm{A} 1$ ou $\mathrm{A} 2$, de poder aquisitivo maior e acesso mais facilitado a bens de consumo.

No presente estudo, a maioria das puérperas adolescentes não trabalhava, portanto, era classificada como fora da população economicamente ativa. Por conseguinte, não possuíam fonte de renda, sendo dependentes da renda do companheiro ou da família. Essa renda, em $64,7 \%$ dos casos, é baixa, e em $51,8 \%$ dos casos as famílias possíam três dependentes. Por serem, em sua maioria, dependentes economicamente do companheiro ou da família, as mães adolescentes necessitam de suporte emocional para vivenciar essa fase da vida.

$\mathrm{O}$ apoio social relaciona-se à dimensão pessoal, sendo constituído por membros de uma rede efetivamente importante ${ }^{(15)}$ para a adolescente. $\mathrm{O}$ apoio recebido pelas puérperas constitui-se, em sua maioria, por laços consanguíneos, portanto, efetivado por pessoas com alguma proximidade. Na gravidez na adolescência destaca-se a importância do apoio familiar, psicológico e social por se constituírem em fatores protetores, minimizando as perdas e desvantagens decorrentes da gravidez precoce. $\mathrm{O}$ apoio, nesse momento da vida das adolescentes, é de fundamental importância para sua vida futura e para a vida dos seus filhos. Pesquisa indica que a reincidência da gravidez na adolescência é menor quando a jovem recebe apoio ${ }^{(16)}$.

No presente estudo, a gravidez na adolescência aparece muito mais como um problema social do que biológico; enfatiza-se a relevância de se ampliar a cobertura e o número de consultas pré-natais, o que poderá contribuir para alcançar melhores resultados com o acompanhamento da gestação, particularmente entre as adolescentes.

Ao assistir o adolescente é primordial a abertura de espaços de escuta e diálogo sobre gravidez, orientando sobre sexualidade, relações de gênero, métodos contraceptivos e DST/Aids. Por se tratar de temas, por vezes constrangedores, deve-se garantir privacidade, confidencialidade e atendimento de qualidade. O profissional deve possuir conhecimentos científicos, técnicos e culturais acerca do tema para que possa orientar os indivíduos a fazerem escolhas conscientes, contribuindo assim para uma paternidade responsável.

\section{CONSIDERAÇÕES FINAIS}

Os resultados demonstram que as características encontradas nos sujeitos, nesta pesquisa, não divergem do que já é encontrado na literatura. As adolescentes que vivenciam a gravidez são, em sua maioria, de cor morena, com baixo nível de escolaridade, alto índice 
de evasão escolar, fora do mercado de trabalho, dependentes economicamente do companheiro ou dos pais, e pertencentes às classes econômicas menos favorecidas e com renda familiar baixa. Essa realidade diminui as chances de ascensão social e contribui para a manutenção do ciclo da pobreza, por dificultar a qualificação profissional e a inserção no mercado de trabalho.

A gravidez na adolescência é um fenômeno complexo que envolve diversos aspectos sociais e que precisa ser trabalhado pelas políticas públicas de saúde e educação, para prevenir sua ocorrência, quando não planejada, bem como, para minimizar as suas consequências biológicas e sociais. Indica-se a realização de novas pesquisas relacionadas a temas que envolvam adolescentes de estratos sociais mais favorecidos, a fim de fazer um comparativo do problema entre as diferentes classes sociais no Brasil.

\section{REFERÊNCIAS}

1. Pantoja ALN. Ser alguém na vida: uma análise sócioantropológica da gravidez/maternidade na adolescência em Belém do Pará - Brasil. Cad Saude Publica. 2003; 19 Supl 2:S335-43.

2. Magalhães MLC, Furtado FM, Nogueira MB, Carvalho FHC, Almeida FML, Mattar R, et al. Gestação na adolescência precoce e tardia: há diferença nos riscos obstétricos? Rev Bras Ginecol Obstet. 2006; 28(8):446-52.

3. Chalem E, Mitsuhiro SS, Ferri CP, Barros MCM, Guinsburg R, Laranjeira R. Gravidez na adolescência: perfil sócio-demográfico e comportamental de uma população da periferia de São Paulo - Brasil. Cad Saúde Pública. 2007; 23(1):177-86.

4. Hoga LAK, Borges ALV, Reberte LM. Razões e reflexos da gravidez na adolescência: narrativas dos membros da família. Esc Anna Nery Rev Enferm. 2010; 14(1):151-7.

5. Holanda ML, Frota MA, Machado MFAS, Vieira NFC. O papel do professor na educação sexual de adolescentes. Cogitare Enferm. 2010; 15(4):702-8.

6. Ximenes Neto FRG, Dias MSA, Rocha J, Cunha ICKU. Gravidez na adolescência: motivos e percepções de adolescentes. Rev Bras Enferm. 200; 60(3):279-85.

7. Yazlle, MEHD. Gravidez na adolescência. Rev Bras Ginecol Obstet. 2007; 28(8):443-5.

8. Meincke SMK. Redes sociais de apoio à paternidade na adolescência. Edital MCT/CNPq/MS-SCTIE-DECIT/
CT-Saúde no 022/2007.

9. Ministério da Sáude(BR). Conselho Nacional de Saúde. Diretrizes e normas regulamentadoras de pesuisa envolvendo seres humanos. Resolução n. 196/96, de 10 de outubro de 1996. Brasília, 1996.

10. Associaão Brasileira de Empresas de Pesquisa (ABEP). Novo Critério Padrão de Classificação Econômica/ Brasil, 2008 [Internet]. São Paulo: UNESP. [acesso em 25 maio 2010]. Disponível: http://www.viverbem.fmb. unesp.br/docs/classificacaobrasil.pdf

11. Faria DGS, Zanetta DMT. Perfil de mães adolescentes de São José do Rio Preto/Brasil e cuidados na assistência pré-natal. Arq Cienc Saude. 2008; 15(1):17-23.

12. Santos GHN, Martins MG, Sousa MS. Gravidez na adolescência e fatores associados com baixo peso ao nascer. Rev Bras Ginecol Obstet. 2008; 30(5):224-31.

13. Ministério da Saúde(BR). [Internet]. Brasília: MS; [acesso em 17 maio 2010]. Pesquisa Nacional de Demografia e Saúde da Criança e da Mulher. Relatório 2008. 2008. Disponível: http://bvsms.saude.gov.br/bvs/ publicacoes/relatorio_pnds_2006.pdf.

14. Ministério da Educação(BR).Ensino fundamental de nove anos - orientações gerais.2004. [acesso em 23 maio 2010]. Disponível: http://portal.mec.gov.br/seb/arquivos/ pdf/Ensfund/noveanorienger.pdf.

15. Pedro ICS, Rocha SMM, Nascimento LC. Apoio e rede social em enfermagem familiar: revendo conceitos. Rev Latino-Am Enfermagem. 2008; 16(2):324-7.

16. Melhado A, Sant'Anna MJC, Passarelli MLB, Coates V. Gravidez na adolescência: apoio integral à gestante e à mãe adolescente como fator de proteção da reincidência. Adolescência e Saúde. 2008; 5(2):45-51. 